\title{
The Natural Way to Tackle Common Health Problems with Fruits and its Benefits
}

\author{
Hameed Mohamed Rafeek* \\ Department of Biomedical Sciences, Sri Lanka
}

*Corresponding author: Hameed Mohamed Rafeek, Department of Biomedical Sciences, Sri Lanka

Received Date: March 05, 2020

Published Date: April 27, 2020

\section{Depression}

Depression is more than just a 'fed up' feeling. It can range from a serious loss of interest in work, relationships and hobbies to becoming a debilitating disease. Most people suffering from depression are aware that they have a problem but those who are not aware may not seek the correct help and symptoms become progressively worse. Depressed individuals may not resort to junk food which is of little nutritional value and comfort eating, or may miss meals altogether because of lack of self interest. The cause of depression is not always clear but its roots may lie in a physical cause such as marital difficulties, unemployment or bereavement which puts undue stress on an individual.

\section{Diet and lifestyle}

Sufferers should consult their doctor to rule out any physical cause. counselling maybe necessary if the problem is anemotional case. It is also important to eat a well balanced diet for this, plus regular exercise can promote feelings of wellbeing.

\section{Useful supplements}

Calcium 500 mg andf magnesium 250 mg- are minerals known to have a calming effect on tense muscles- typical in stressed individuals. High potency vitamin $\mathrm{b}$ complex $\mathrm{b}$ vitamins are required for healthy nerves and a healthy nervous system.

Vitamin C 1 gram three times daily- vitamin C levels aare easily depleted during periods of stress.

\section{Headache and migraine}

There are typically three categories of headaches-tension, cluster and migraine. Recurrent headaches are known as cluster headaches. With migraine there is a deep throbbing pain which can be accompanied by other symptoms such as blurred vision, which can affect the person for several hours or days. Most headaches are caused by muscular tension which causes blood vessels to spasm but no one is really sure of the underlying causes of migraine.

\section{Diet and lifestyle}

Try to ensure that you take regular breaks throughout the day especially if you are working with a computer. During this time try to relax by using breathing techniques. Regular exercise is a good way to encourage the body to release tension. Avoid stimulants such as tea and coffee but try to include 6-8 glasses of water a day to help eliminate toxins from the body.

\section{Useful supplements}

Magnesium $500 \mathrm{mg}$ and calcium $1000 \mathrm{mg}$ daily for muscle health. Kava kava 400mg extract daily to aid the body in relaxing.

\section{Other supplements to consider}

Ginger 120 mg extract daily -studies have shown promising effects when ginger is used by migraine sufferers.

Vitex angus castus $100 \mathrm{mg}$ extract daily for when headaches appear to occur premenstrually.

\section{Acknowledgement}

None.

\section{Conflicts of Interest}

No conflicts of interest. 